\title{
ANALYSIS OF DRUG DISSOLUTION DATA
}

\author{
JACK C. LEE ${ }^{1 *}$, DUNG-TSA CHEN ${ }^{1}$, HUI-NIEN HUNG ${ }^{1}$ AND JAMES J. CHEN ${ }^{2}$ \\ ${ }^{1}$ Institute of Statistics, National Chiao Tung University, Hsinchu, Taiwan \\ ${ }^{2}$ Division of Biometry and Risk Assessment, National Center for Toxicological Research, Jefferson, Arkansas 72079, U.S.A.
}

\begin{abstract}
SUMMARY
Drug absorption in the human body depends on the dissolution rate of the drug. Suitable dissolution characteristics are important to ensure that the drug will achieve the desired therapeutic effects. To assess the similarity of dissolution rates of several drug lots, we apply a general growth curve model with different covariance structures. The Box-Cox power transformation and the naive log transformation are applied to a function of the dissolution rate. The predictive sample-reuse, or cross-validation, method is employed in selecting an appropriate model with best predictive accuracy. A testing procedure for examining the similarity among the drug lots is also conducted. A partially Bayesian approach is used for the assessment of dissolution equivalence. Copyright (C) 1999 John Wiley \& Sons, Ltd.
\end{abstract}

\section{INTRODUCTION}

The United States Food and Drug Administration (FDA) regulation requires dissolution testing, when applicable, to confirm that the dissolution profile is consistent with the product which formed the basis of new drug application (NDA) approval. Such a requirement is to be met when a new lot is produced as well as during the shelf life stability estimation. The dissolution testing apparatus is typically constructed so that dissolution testing may be performed on sampling units of six tablets or capsules simultaneously. The dissolution value of an active ingredient is measured at various predetermined time intervals to perform a dissolution profile analysis. For immediate release drug products that dissolve almost completely within 30 minutes, the specification is typically verified with the average dissolution value, plus or minus 2 to 3 standard deviations, at 15 or 30 minutes of the tested tablets of initial batches. For drug products that have slower dissolution rates, the dissolution profile is determined by dissolution values at multiple time points. The specifications of the sampling acceptance criteria are published in the U.S. Pharmacopoeia (USP) and are often used by the pharmaceutical industry as the standard for the innovative and generic drug products.

For the drug dissolution data, as given in Table I, for each tablet, the dissolution rates have been measured at seven time points: 1, 2, 3, 4, 6, 8 and 10 minutes. Our interest is to compare the dissolution curves between the test lot and the standard lots.

\footnotetext{
* Correspondence to: Jack C. Lee, Institute of Statistics, National Chiao Tung University, Hsinchu, Taiwan

Contract/grant sponsor: National Science Council of Taiwan

Contract/grant number: 87-2119-M-009-001 and 86-2115-M-009-037-T
}

CCC 0277-6715/99/070799-16\$17.50

Copyright (C) 1999 John Wiley \& Sons, Ltd.
Received January 1998 Accepted June 1998 
Table I. Dissolution rates of test and standard lots

\begin{tabular}{|c|c|c|c|c|c|c|c|}
\hline Time point & 1 & 2 & 3 & 4 & 5 & 6 & 7 \\
\hline \multirow[t]{12}{*}{ Lot 1} & 50 & 56 & 68 & 73 & 80 & 86 & 87 \\
\hline & 43 & 48 & 65 & 71 & 77 & 85 & 92 \\
\hline & 44 & 54 & 63 & 67 & 74 & 81 & 82 \\
\hline & 48 & 56 & 64 & 70 & 81 & 84 & 93 \\
\hline & 45 & 56 & 63 & 69 & 76 & 81 & 83 \\
\hline & 46 & 57 & 64 & 67 & 76 & 79 & 85 \\
\hline & 42 & 56 & 62 & 67 & 73 & 81 & 88 \\
\hline & 44 & 54 & 60 & 65 & 72 & 77 & 83 \\
\hline & 38 & 46 & 54 & 58 & 66 & 70 & 76 \\
\hline & 46 & 55 & 63 & 65 & 73 & 80 & 85 \\
\hline & 47 & 55 & 62 & 67 & 76 & 81 & 85 \\
\hline & 48 & 55 & 62 & 66 & 73 & 78 & 85 \\
\hline \multirow[t]{12}{*}{ Lot 2} & 48 & 57 & 67 & 73 & 80 & 85 & 87 \\
\hline & 47 & 58 & 65 & 71 & 75 & 82 & 87 \\
\hline & 45 & 52 & 61 & 66 & 73 & 81 & 84 \\
\hline & 37 & 51 & 60 & 71 & 81 & 84 & 91 \\
\hline & 43 & 56 & 63 & 69 & 76 & 81 & 85 \\
\hline & 48 & 56 & 64 & 65 & 73 & 79 & 82 \\
\hline & 36 & 49 & 61 & 67 & 73 & 81 & 81 \\
\hline & 41 & 54 & 61 & 65 & 70 & 77 & 82 \\
\hline & 38 & 41 & 54 & 58 & 64 & 70 & 77 \\
\hline & 45 & 55 & 61 & 65 & 72 & 80 & 83 \\
\hline & 48 & 55 & 63 & 67 & 74 & 81 & 83 \\
\hline & 44 & 55 & 61 & 66 & 74 & 78 & 82 \\
\hline \multirow[t]{12}{*}{ Lot 3} & 47 & 56 & 67 & 73 & 81 & 86 & 88 \\
\hline & 45 & 46 & 65 & 73 & 77 & 81 & 90 \\
\hline & 45 & 54 & 62 & 67 & 74 & 81 & 81 \\
\hline & 35 & 46 & 59 & 70 & 81 & 84 & 90 \\
\hline & 48 & 51 & 63 & 68 & 74 & 81 & 83 \\
\hline & 48 & 52 & 64 & 67 & 72 & 79 & 87 \\
\hline & 46 & 51 & 62 & 63 & 73 & 81 & 84 \\
\hline & 46 & 54 & 61 & 65 & 72 & 75 & 86 \\
\hline & 38 & 46 & 54 & 58 & 66 & 70 & 76 \\
\hline & 41 & 55 & 61 & 65 & 76 & 80 & 86 \\
\hline & 42 & 55 & 62 & 65 & 76 & 85 & 85 \\
\hline & 48 & 51 & 62 & 63 & 74 & 78 & 84 \\
\hline \multirow[t]{12}{*}{ Test Lot } & 34 & 45 & 61 & 66 & 75 & 85 & 91 \\
\hline & 36 & 51 & 62 & 67 & 83 & 85 & 93 \\
\hline & 37 & 48 & 60 & 69 & 76 & 84 & 91 \\
\hline & 35 & 51 & 63 & 61 & 79 & 82 & 88 \\
\hline & 36 & 49 & 62 & 68 & 79 & 81 & 89 \\
\hline & 37 & 52 & 65 & 73 & 82 & 93 & 95 \\
\hline & 39 & 51 & 61 & 69 & 77 & 85 & 93 \\
\hline & 38 & 49 & 63 & 66 & 79 & 84 & 90 \\
\hline & 35 & 51 & 61 & 67 & 80 & 88 & 96 \\
\hline & 37 & 49 & 61 & 68 & 79 & 91 & 91 \\
\hline & 37 & 51 & 63 & 71 & 83 & 89 & 94 \\
\hline & 37 & 54 & 64 & 70 & 80 & 90 & 93 \\
\hline
\end{tabular}


For multiple time-point dissolution specifications the conventional application is to apply the USP plan independently at each individual time point. This approach does not take into account the correlations among dissolution rates at various time points. Chen and Tsong ${ }^{1}$ proposed using multivariate confidence regions of the dissolution values at each time point. However, they failed to take into consideration the fact that dissolution rate changes with time.

For extended release drug products, the dissolution profile for the whole time course is often of interest; thus, it may be preferable to establish the specifications based on the dissolution curve which is a function of time. This paper proposes a growth curve coupled with the Box-Cox transformation to model the dissolution curves. The proposed methods are based on a regression model for the dissolution rates with the time of observations as the independent variable. Also, the Box-Cox transformation will help enhance the validity of the model assumptions. Two methods are proposed for the lot acceptance. One is based on the confidence region of the coefficients of the fitted dissolution curve. The other is based on the likelihood ratio test when the covariance matrix is arbitrary positive definite. A partially Bayesian approach for the assessment of dissolution equivalence is also given.

\section{EXAMPLE}

The data given in Table I are the dissolution rates of three standard lots and one test lot. For each lot, there are twelve tablets and for each tablet the dissolution rates are measured at seven time points: 1, 2, 3, 4, 6, 8 and 10 minutes. Our interest is to compare the dissolution curves between the test lot and the standard lots. The dissolution function $F(t)$ of a drug is defined to be the percentage of a tablet that has dissolved at time $t$, and $R(t)$ is defined by $F(t) /(1-F(t))$. Since $0 \leqslant F(t) \leqslant 1$, and $R(t) \geqslant 0$, both ranges are not the entire real line. It may cause the out of range problem when we model them directly. We will therefore consider applying the Box-Cox and log transformations to $R(t)$ which will avoid the above problem.

To find an appropriate drug dissolution profile curve from the standard lots, scatter plots of $\log R(t)$ in Figure 1 show that it is not a simple linear function of time $t$. There are two possible change points at time 3 and time 6 , because the slopes of the curves change significantly at these two time points. Plot of $F(t)$ and $R(t)$ give somewhat clearer pictures of the non-linear relationships.

Hence, for each lot, a piecewise linear regression model for the drug dissolution profile curve seems to be more appropriate for this data set. This can be handled by the well-known growth curve model of Potthoff and Roy. ${ }^{2}$ Also the famous Box-Cox transformation is useful for this type of data, as explained later. Thus, in order to model the drug dissolution profile curve properly, we will use the growth curve model with the Box-Cox transformation which will be discussed in Section 3. The proposed model will handle this type of data nicely.

\section{METHODS}

We will next discuss some methods for dealing with the drug dissolution data as shown in Table I.

\subsection{Modelling the Dissolution Curve}

From Figure 1, it is clear that $\log F /(1-F)$ is not quite linear, but piecewise linear, in time. The famous Box-Cox transformation applied to $F /(1-F)$ will help achieve the linearity property. It 

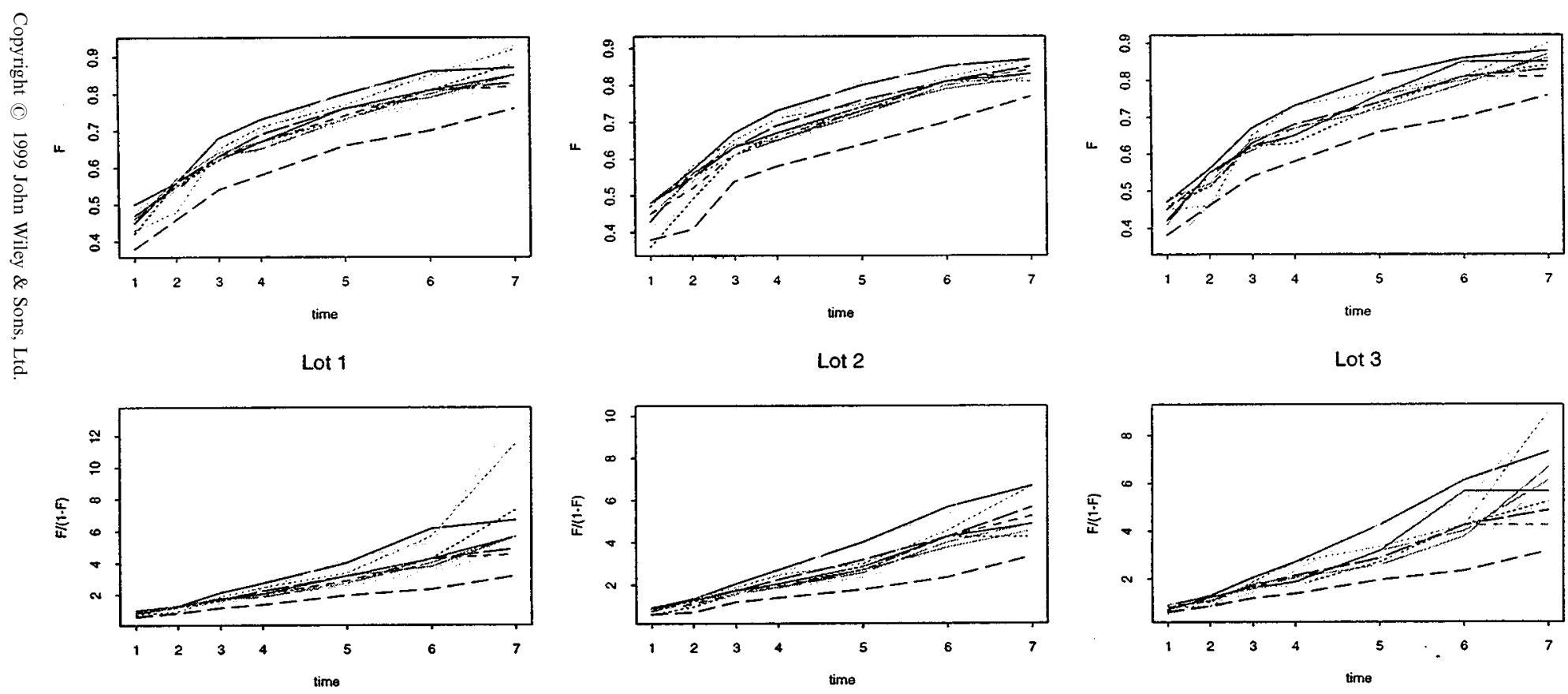

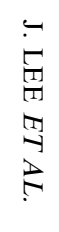

Lot 1
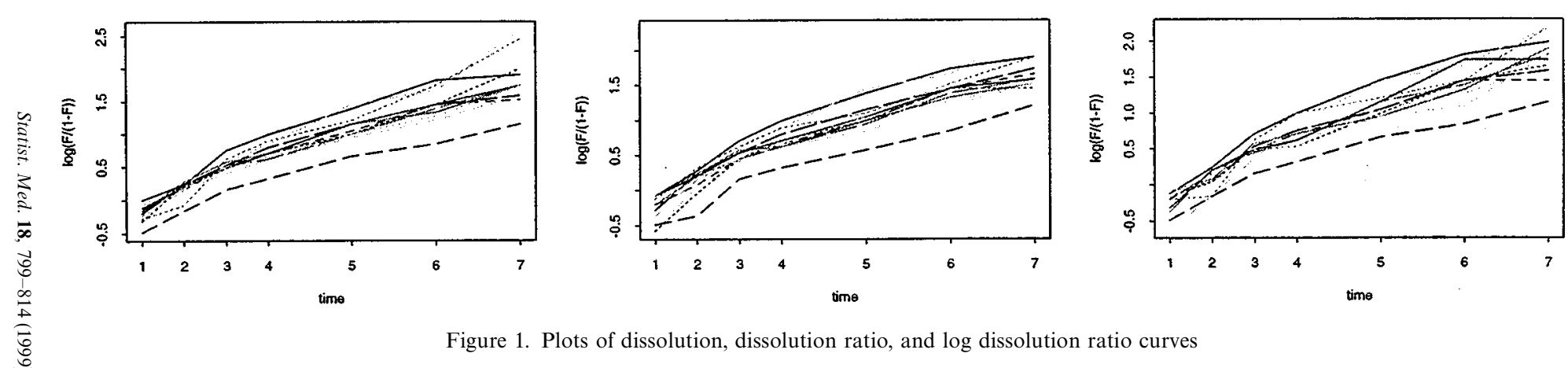

Figure 1. Plots of dissolution, dissolution ratio, and log dissolution ratio curves 
is noted that the Box-Cox transformation will include the log transformation as a special case. For this reason, we apply the Box-Cox transformation to the growth curve model. More specifically, let $Y_{i j}$ be a function of observed drug dissolution rate at the $i$ th time for the $j$ th tablet. The Box-Cox transformation of $Y_{i j}$ is defined as

$$
Y_{i j}^{(\lambda)}= \begin{cases}\frac{\left(Y_{i j}+v\right)^{\lambda}-1}{\lambda} & \text { if } \lambda \neq 0 \\ \log \left(Y_{i j}+v\right) & \text { if } \lambda=0\end{cases}
$$

where $v$ is a known constant such that $Y_{i j}+v>0$.

If $Y_{i j}^{(\lambda)}$ is a linear or piecewise linear function of time, then putting in a matrix form, $Y^{(\lambda)}=\left(Y_{1}^{(\lambda)}, Y_{2}^{(\lambda)}, \ldots, Y_{N}^{(\lambda)}\right)$, where $Y_{j}^{(\lambda)}=\left(Y_{1 j}^{(\lambda)}, Y_{2 j}^{(\lambda)}, \ldots, Y_{p j}^{(\lambda)}\right)^{\prime}$, can be represented as a growth curve model defined in (2) of the Appendix.

With regard to the covariance matrix $\Sigma$, specified in (2), due to the lack of any structural information, we will assume $\Sigma$ being either arbitrary positive definite, which is the most general situation, or with an $\operatorname{AR}(1)$ dependence, which will usually be a useful first approximation for somewhat short and serially observed data.

\subsection{Parameter Estimation}

For the general growth curve model as specified in (2), the maximum likelihood estimates of the parameters $\tau, \Sigma$ and $\lambda$ when $\Sigma$ is assumed arbitrary positive definite and $\tau, \rho, \sigma^{2}$ and $\lambda$ when $\Sigma$ is assumed to have an AR(1) dependence are given in the Appendix. The computations involved are relatively simple and should present no difficulty in practice.

\subsection{Model Selection}

In this subsection we will address the issue of the model selection with respect to the covariance matrix $\Sigma$. As noted earlier, we assume two covariance structures for $\Sigma$, one being arbitrary positive definite and the other being an AR(1) dependence. The likelihood ratio test and a predictive accuracy procedure are discussed here.

Lee $^{3}$ proposed a likelihood ratio test procedure for testing AR(1) covariance structure, that is,

$$
H_{0}: \Sigma=\sigma^{2} C \text { versus } H_{1}: \Sigma \text { is arbitrary positive definite }
$$

where $C=\left(\rho^{|i-j|}\right)$.

Under $H_{0}$, we have $-2 \ln \Lambda \sim \chi_{v}^{2}$, as $N \rightarrow \infty$, where $v=\frac{p(p+1)}{2}-2$ and $\Lambda$ is the likelihood ratio statistic as defined in (5).

The statistic, as given in (5), is easy to compute because it involves only simple matrix computations which are available in most software such as SAS and S-plus. However, acceptance of a certain null hypothesis does not mean that other hypotheses are not suitable for the data. Alternatively, a conditional prediction approach via the predictive sample reuse (PSR) method of Geisser ${ }^{4}$ can be used for selecting an appropriate model for the growth curve data. We will next discuss the conditional prediction for the general growth curve model.

After observing the sample $Y$, some partial observations on $V$, namely $V^{(1)}$, are also at hand, and our interest is in predicting $V^{(2)}$ given $Y$ and $V^{(1)}$ where

$$
V=\left(\begin{array}{l}
V^{(1)} \\
V^{(2)}
\end{array}\right)
$$

$V$ is $p \times 1, V^{(i)}$ is $p_{i} \times 1$ and $p_{1}+p_{2}=p$.

Lee and Geisser ${ }^{5}$ suggested the predictor $\hat{V}^{(2)}$ of $V^{(2)}$ as given in (6). 
In order to select an appropriate covariance structure of $\Sigma$, we can utilize the observed sample $Y$ to do the conditional prediction of $V^{(2)}$ given $V^{(1)}$ repeatedly in the following manner. Let $Y=\left(Y_{1}, \ldots, Y_{N}\right)$ and $Y_{i}=\left(Y_{i}^{(1)^{\prime}}, Y_{i}^{(2)^{\prime}}\right)^{\prime}$ where $Y_{i}^{(1)}$ is $p_{1} \times 1$ and $Y_{i}^{(2)}$ is $p_{2} \times 1$. In the PSR method, $Y_{i}^{(2)}$ is treated as the unobserved portion of the 'future' vector $V^{(2)}$ and $Y_{i}^{(1)}$ the partially observed portion. $Y_{i}^{(2)}$ is then predicted using (6) with the sample $Y_{(i)}=\left(Y_{1}, Y_{2}, \ldots, Y_{i-1}, Y_{i+1}, \ldots, Y_{N}\right)$. Thus, each vector $Y_{j}$ is included in the sample $N-1$ times in the prediction process, and hence the procedure is called the PSR method.

The discrepancy measure by the PSR method is defined by the mean absolute relative deviation (MARD) of the predicted values $\hat{V}^{(2)}$ from the actual observation $V^{(2)}$, defined as $\frac{1}{p_{2} N} \Sigma_{i=1}^{N} \Sigma_{j=p_{1}+1}^{p}\left|\left(\widehat{V_{i j}^{(2)}}-V_{i j}^{(2)}\right) / V_{i j}^{(2)}\right|$. The selected model corresponds to the minimum MARD. This procedure is a data analytic aparametric method which simulates the predictive process within the sample, given a complete lack of any distributional assumption. It can also be applied to any number of competing models, nested or not. One drawback is computer-consumed. For further details, please refer to Lee $^{3}$ and Keramidas and Lee. ${ }^{6}$

\subsection{Testing Equality of Mean Functions when $\Sigma$ is Arbitrary Positive Definite}

When an appropriate model is chosen, there are two types of problems to be investigated. One is the one sample problem, the other is the $r$ samples problem for $r \geqslant 2$. The procedures, adapted from $\mathrm{Khatri}^{7}$ and given in the Appendix, are based on the likelihood ratio criterion when the covariance matrix $\Sigma$ is arbitrary positive definite.

The one sample test is for testing the hypothesis, $H_{0}: \tau=\tau_{0}$, versus $H_{1}: \tau \neq \tau_{0}$, and we use the statistic $Q\left(\tau_{0}\right)$ as given in (7).

The $r$ sample test is designed for testing that the $r$ mean functions are similar, that is,

$$
H_{0}: \tau D=\mathbf{0}, \text { versus } H_{1}: \tau D \neq \mathbf{0}
$$

where $\tau=\left(\tau_{1}, \tau_{2}, \ldots, \tau_{r}\right)$ and

$$
\underset{r \times q}{D}=\left(\begin{array}{rrcr}
1 & 0 & \cdots & 0 \\
-1 & 1 & 0 & \vdots \\
0 & -1 & \ddots & 0 \\
\vdots & 0 & \ddots & 1 \\
0 & \ldots & 0 & -1
\end{array}\right) .
$$

We will use the statistic $Q(\tau D=0)$, as given in (9), and the critical values are given in Krishnaiah and Lee. ${ }^{8}$

\subsection{Dissolution Equivalence}

We next consider testing the null hypothesis $H_{0}=\left\{\theta=\left(\beta, \beta_{0}, \Sigma\right):\left|\beta_{i}-\beta_{0, i}\right|>\delta\left|\beta_{0, i}\right|\right.$ for some $i\}$ versus the alternative hypothesis $H_{1}=\left\{\theta=\left(\beta, \beta_{0}, \Sigma\right):\left|\beta_{i}-\beta_{0, i}\right| \leqslant \delta\left|\beta_{0, i}\right|\right.$ for all $\left.i\right\}$, where $\beta$ and $\beta_{0}$ are $m \times 1$ mean vectors, $\delta$ is a real number between 0 and 1 , and $\Sigma$ is a $p \times p$ covariance matrix. It is noted that $100 \delta$ per cent is the percentage of the relative difference between $\beta_{i}$ and $\beta_{0, i}$. The equivalence between the test and standard lots is concluded if $H_{1}$ is accepted. The purpose is to find the likelihood function of $H_{0}, L\left(H_{0}\right)$, versus that of $H_{1}, L\left(H_{1}\right)$. The main idea is 
to use the averaging as follows:

$$
\begin{aligned}
& L\left(H_{0}\right)=\int_{R_{1}} L(\theta, x) \pi_{1}(\theta) \mathrm{d}(\theta) \\
& L\left(H_{1}\right)=\int_{R_{2}} L(\theta, x) \pi_{2}(\theta) \mathrm{d}(\theta)
\end{aligned}
$$

where $R_{1} \subset H_{0}, R_{2} \subset H_{1}, \pi_{1}$ is a probability measure on $R_{1}$, and $\pi_{2}$ is a probability measure on $R_{2}$. Thus the problem boils down to the choosing of $R_{1}, R_{2}, \pi_{1}$ and $\pi_{2}$. From the idea of average likelihood, ${ }^{9}$ we will choose $\pi_{i}(\theta) \mathrm{d}(\theta) \propto|\Sigma|^{\frac{p+1}{2}} \mathrm{~d}\left(\Sigma^{-1}\right) \mathrm{d}\left(\beta_{0}\right) \mathrm{d}(\beta)$ for $i=1$, 2. Since the range of $\beta$ in $H_{1}$ is bounded, usually we can choose $R_{2}$ to be $H_{1}$, but the range of $\beta$ in $H_{0}$ is unbounded, and we will have some trouble. From simulation, we suggest choosing $R_{1}=\left\{\theta=(\beta, \Sigma):\left|\beta_{i}-\hat{\beta}_{0, i}\right|\right.$ $\leqslant a\left|\hat{\beta}_{0, i}\right|$ for all $\left.i\right\}$ where ' $a$ ' satisfies Volume $\left(\left\{\beta:\left|\beta_{i}-\hat{\beta}_{0, i}\right| \leqslant a\left|\hat{\beta}_{0, i}\right|\right\}-\left\{\beta:\left|\beta_{i}-\hat{\beta}_{0, i}\right| \leqslant\right.\right.$ $\left.\left.\left.\delta\left|\hat{\beta}_{0, i}\right|\right\}\right)=\operatorname{Volume}\left\{\beta:\left|\beta_{i}-\hat{\beta}_{i}\right| \leqslant \delta\left|\hat{\beta}_{i}\right|\right\}\right)$ and is close to $2^{1 / m}$ in general. Therefore we can calculate the likelihood functions $L\left(H_{0}\right)$ and $L\left(H_{1}\right)$, and the Bayes factor $=\frac{L\left(H_{0}\right)}{L\left(H_{1}\right)}$. The equivalence is established if the Bayes factor is less than 1. If we have some priors $\pi\left(H_{0}\right)$ and $\pi\left(H_{1}\right)$ on $H_{0}$ and $H_{1}$, we can easily get the posterior odds, $\frac{L\left(H_{0}\right) \pi\left(H_{0}\right)}{L\left(H_{1}\right) \pi\left(H_{1}\right)}$, and then use this odds to perform the test.

\section{RESULTS}

In this section we will apply the results of the previous section to the drug dissolution data in Section 1. As discussed in Section 2, both Box-Cox and the $\log$ transformations will be considered for this data set. It is noted that both transformations, when applied to $R(t)$ for the pooled data, can stabilize the variance, as shown in Table II. Also, since there are two possible change points at time 3 and time 6 , the design matrix $X$ for the growth curve model is

$$
X=\left(\begin{array}{lllllll}
1 & 1 & 1 & 1 & 1 & 1 & 1 \\
1 & 2 & 3 & 3 & 3 & 3 & 3 \\
0 & 0 & 0 & 1 & 3 & 5 & 5 \\
0 & 0 & 0 & 0 & 0 & 0 & 2
\end{array}\right)^{\prime}
$$

and the model can be written as $Y_{i j}=\beta_{0}+\beta_{1} x_{1}+\beta_{2} x_{2}+\beta_{3} x_{3}+\varepsilon_{i, j}$, for $i=1, \ldots, p$; $j=1, \ldots, N$,

$$
x_{1}=\left\{\begin{array}{ll}
t & \text { for } 0 \leqslant x_{1}<3 \\
3 & x_{1} \geqslant 3
\end{array}, \quad x_{2}=\left\{\begin{array}{cc}
0 & \text { for } x_{2}<3 \\
t-3 & 3 \leqslant x_{2}<8 \\
5 & x_{2} \geqslant 8
\end{array}, \quad x_{3}=\left\{\begin{array}{cc}
0 & \text { for } x_{3}<8 \\
t-8 & 8 \leqslant x_{3}<10 \\
2 & x_{3} \geqslant 10
\end{array}\right.\right.\right.
$$

and $Y_{i j}$ could be $F(t), R(t)$, or Box-Cox transformed $F(t)$ (or $R(t)$ ) at the $i$ th time point for $j$ th tablet.

To compare the Box-Cox and log transformations, the prediction performance of the predictor as given in (6), in terms of MARD, as described in Section 3.3, is considered. Sine $p_{2}$ has to be specified in the prediction of $V^{(2)}$, a simulation of prediction performance for data generated from the $\operatorname{AR}(1)$ dependence structure $(N=12, p=7, m=4)$ with 300 replications is conducted and reported in Table III. When $p_{2} \leqslant 4$, the mean of the difference in MARDs between arbitrary positive definite covariance matrix and $\mathrm{AR}(1)$ covariance matrix is more than seven times of the 
Table II. Sample variances for different transformations of $R(t)$ for pooled data

\begin{tabular}{lccccccc}
\hline Time point & 1 & 2 & 3 & 4 & 5 & 6 & 7 \\
\hline$R(t)$ & 0.015 & 0.028 & 0.044 & 0.124 & 0.413 & 0.937 & $4 \cdot 851$ \\
$\log R(t)$ & 0.026 & 0.026 & 0.018 & 0.031 & 0.045 & 0.059 & 0.105 \\
power $(0 \cdot 7)$ & 0.017 & 0.027 & 0.033 & 0.081 & 0.210 & 0.402 & $1 \cdot 487$ \\
Powers & & & & & & & \\
$\left(\hat{\lambda}_{1}, \hat{\lambda}_{2}, \hat{\lambda}_{3}\right)^{*}$ & 0.029 & 0.026 & 0.015 & 0.015 & 0.014 & 0.018 & 0.020 \\
\hline$* \hat{\lambda}_{1}=-0.179$ is applied to time points 1, 2 and 3 & & & & & \\
$\hat{\lambda}_{2}=-0.553$ is applied to time points 4 and 5 & & & & & \\
$\hat{\lambda}_{1}=-0.459$ is applied to time points 6 and 7 & & & & &
\end{tabular}

Table III. Prediction performance for data generated from AR(1) structure with 300 replications*

\begin{tabular}{llllllll}
\hline MARD & $V_{7}$ & $V_{6}-V_{7}$ & $V_{5}-V_{7}$ & $V_{4}-V_{7}$ & $V_{3}-V_{7}$ & $V_{2}-V_{7}$ & $V_{1}-V_{7}$ \\
\hline ar1 & 0.063 & 0.080 & 0.102 & 0.162 & 0.325 & 0.768 & $2 \cdot 125$ \\
arb & 0.102 & 0.117 & 0.134 & 0.199 & 0.351 & 0.815 & $2 \cdot 158$ \\
arb-ar1 & 0.039 & 0.037 & 0.031 & 0.037 & 0.026 & 0.046 & 0.033 \\
SE of (arb-ar1) & 0.002 & 0.002 & 0.001 & 0.005 & 0.009 & 0.021 & 0.047 \\
\% of (arb-ab1 $>0)$ & 0.91 & 0.95 & 0.93 & 0.92 & 0.81 & 0.74 & 0.54 \\
\hline
\end{tabular}

$* V_{j}-V_{7}$ denotes the last $7-j+1$ values of $V$

corresponding standard error (SE). It is therefore safe to claim that the predictive performance for the AR(1) covariance matrix is better. Also, the percentage that the AR(1) covariance performance is better is at least 91 per cent.

From the simulation results given in Table III, it is reasonable to set $p_{2}=1,2,3,4$ in the conditional prediction process. Furthermore, for the likelihood ratio test statistic (8) with $\alpha=0.05$ the acceptance rate reaches only 63 per cent which is much less than the theoretical rate of 95 per cent. Thus, the likelihood ratio test could give a misleading result in small samples.

The comparison in prediction performance using both transformations is summarized in Table IV and Figure 2. The Box-Cox transformation always gives smaller MARDs than the log transformation for three standard lots. This is true for both arbitrary positive definite and AR(1) covariance matrices. However, since their differences are quite small and the $\log R(t)$ is easier to compute and interpret, the following analyses are based on this transformation.

Pooled data always contains more information than single lots if they came from the same population. Furthermore, the three standard lots have similar performance and similar scatter plots. Thus, the comparison of predictive performance is considered for both pooled data and single lots. The pooled data are used to estimate the parameters and then to compute the MARD for each lot. Table V and Figure 3 summarize the prediction results. The performances of pooled data are always better than those of individual lots. Moreover, the arbitrary positive definite covariance matrix has better performance than the AR(1) covariance. Hence, the three single lots are combined for analyses and arbitrary positive definite covariance is selected as the covariance structure. 
Table IV. Prediction performance of different models*

\begin{tabular}{|c|c|c|c|c|c|c|}
\hline \multirow[t]{2}{*}{ Drug data } & \multirow[t]{2}{*}{$\Sigma$} & \multirow[t]{2}{*}{ Transformation } & \multicolumn{4}{|c|}{ MARD $(0 \cdot 1 \%)$} \\
\hline & & & $V_{7}$ & $V_{6}-V_{7}$ & $V_{5}-V_{7}$ & $V_{4}-V_{7}$ \\
\hline Lot 1 & $\begin{array}{c}\text { arbitrary } \\
\text { positive definite } \\
\text { AR(1) }\end{array}$ & $\begin{array}{c}\log \\
\text { power } \\
\text { log } \\
\text { power }\end{array}$ & $\begin{array}{l}83 \cdot 4 \\
80 \cdot 5 \\
28 \cdot 9 \\
27 \cdot 7\end{array}$ & $\begin{array}{l}47 \cdot 9 \\
46 \cdot 9 \\
29 \cdot 8 \\
29 \cdot 4\end{array}$ & $\begin{array}{l}32 \cdot 0 \\
31 \cdot 3 \\
27 \cdot 1 \\
25 \cdot 8\end{array}$ & $\begin{array}{l}31 \cdot 5 \\
29 \cdot 2 \\
30 \cdot 0 \\
26 \cdot 9\end{array}$ \\
\hline Lot 2 & $\begin{array}{c}\text { arbitrary } \\
\text { positive definite } \\
\text { AR(1) }\end{array}$ & $\begin{array}{c}\log \\
\text { power } \\
\text { log } \\
\text { power }\end{array}$ & $\begin{array}{l}41 \cdot 2 \\
39 \cdot 2 \\
16 \cdot 6 \\
16 \cdot 9\end{array}$ & $\begin{array}{l}29 \cdot 4 \\
28 \cdot 3 \\
17 \cdot 4 \\
16 \cdot 7\end{array}$ & $\begin{array}{l}29 \cdot 1 \\
25 \cdot 7 \\
22 \cdot 0 \\
19 \cdot 9\end{array}$ & $\begin{array}{l}38 \cdot 4 \\
36 \cdot 8 \\
30 \cdot 1 \\
27 \cdot 4\end{array}$ \\
\hline Lot 3 & $\begin{array}{c}\text { arbitrary } \\
\text { positive definite } \\
\text { AR(1) }\end{array}$ & $\begin{array}{l}\log \\
\text { power } \\
\text { log } \\
\text { power }\end{array}$ & $\begin{array}{l}41 \cdot 0 \\
40 \cdot 9 \\
34 \cdot 7 \\
35 \cdot 1\end{array}$ & $\begin{array}{l}36 \cdot 2 \\
34 \cdot 6 \\
30 \cdot 4 \\
30 \cdot 4\end{array}$ & $\begin{array}{l}35 \cdot 4 \\
32 \cdot 5 \\
34 \cdot 4 \\
34 \cdot 9\end{array}$ & $\begin{array}{l}30 \cdot 9 \\
29 \cdot 4 \\
38 \cdot 4 \\
38 \cdot 6\end{array}$ \\
\hline
\end{tabular}

$* V_{j}-V_{7}$ denotes the last $7-j+1$ values of $V$

Next, the testing procedures discussed in Section 3.4 are applied to the drug dissolution data for testing four hypotheses. The $\tau$ estimates are given in Table VI.

The first test is a one sample test. The purpose is to check if the mean function of a test lot is equal to the standard which is assumed known. Here we use all three standard lots as the standard. By applying (8) with $m=4, N=12, p=7, \tau_{0}=(-0.575,0.357,0 \cdot 180,0 \cdot 143)^{\prime}$, $\hat{\tau}=(-1 \cdot 069,0 \cdot 519,0 \cdot 275,0 \cdot 283)^{\prime}$, we have the statistic $F_{1}=55 \cdot 35>F_{4,5}(0 \cdot 95)=5 \cdot 19$. We conclude that the test lot's mean function is not equal to the standard.

Another test is a two sample test which is a comparison between test lot and the combined standard lots. Here both mean functions have to be estimated. Applying (9) and (10) with $D=(1,-1)^{\prime}$, we have, under $H_{0}, Q(\tau D=0) \sim U_{1,4,40}$ and the statistic $F_{2}=30 \cdot 63>$ $F_{4,40}(0 \cdot 95)=2 \cdot 61$. The null hypothesis is rejected at the 5 per cent level, which means the test lot does not meet the specification.

The third test is a four sample test which compares the test lot with the three standard lots. Applying (9) with

$$
D=\left(\begin{array}{rrr}
1 & 0 & 0 \\
-1 & 1 & 0 \\
0 & -1 & 1 \\
0 & 0 & -1
\end{array}\right)
$$

we have, under $H_{0}, Q(\tau D=0) \sim U_{3,4,42}$. Comparing with the critical value given in Krishnaiah and Lee, ${ }^{8}$ we reject the null hypothesis at the 5 per cent level, which means that the test lot is different from the three standard lots.

The last test is a test of homogeneity among the three standard lots. Here, $q=2, N=36, r=3$, $p=7, m=4$ and the estimates of the three mean functions are $\hat{\tau}_{1}=(-0 \cdot 564,0 \cdot 362,0 \cdot 179,0 \cdot 174)^{\prime}$, 

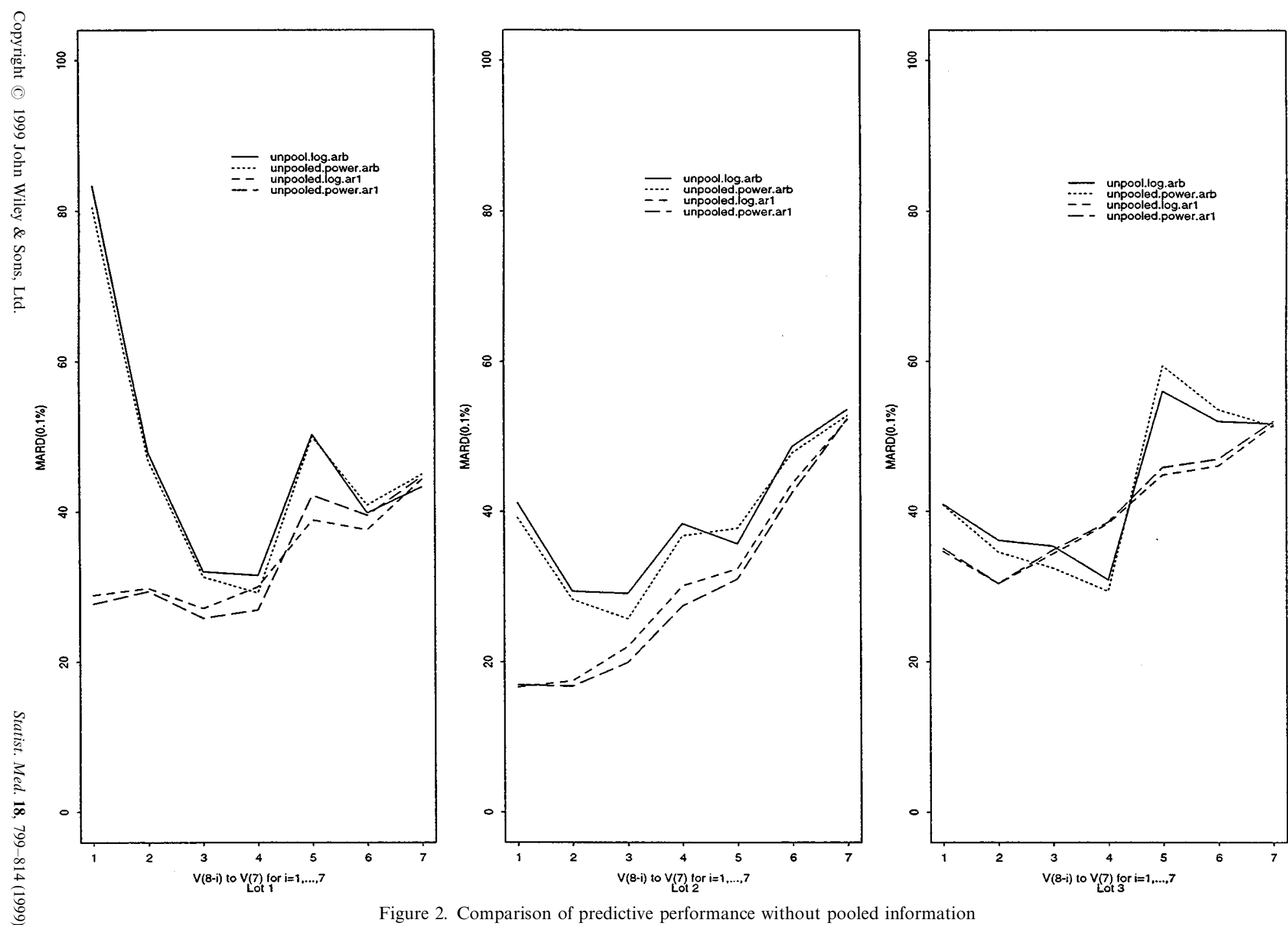
Table V. Prediction performance of different models with pooled data and log transformation

\begin{tabular}{lccccc}
\hline Drug data & $\Sigma$ & \multicolumn{4}{c}{ MARD $(0 \cdot 1 \%)$} \\
\cline { 3 - 6 } & & $V_{7}$ & $V_{6}-V_{7}$ & $V_{5}-V_{7}$ & $V_{4}-V_{7}$ \\
\hline \multirow{2}{*}{ Lot 1} & arbitrary & $31 \cdot 5$ & $21 \cdot 1$ & $20 \cdot 1$ & $23 \cdot 3$ \\
& AR(1) & $25 \cdot 2$ & $26 \cdot 4$ & $24 \cdot 5$ & $27 \cdot 8$ \\
Lot 2 & arbitrary & $20 \cdot 2$ & $16 \cdot 9$ & $21 \cdot 3$ & $28 \cdot 1$ \\
& AR(1) & $23 \cdot 6$ & $21 \cdot 4$ & $24 \cdot 4$ & $30 \cdot 7$ \\
Lot & arbitrary & $23 \cdot 5$ & $22 \cdot 4$ & $29 \cdot 8$ & $24 \cdot 3$ \\
& AR(1) & $31 \cdot 2$ & $25 \cdot 3$ & $29 \cdot 5$ & $33 \cdot 7$ \\
\hline
\end{tabular}

* $V_{j}-V_{7}$ denotes the last $7-j+1$ values of $V$

$\hat{\tau}_{2}=(-0 \cdot 570,0 \cdot 348,0 \cdot 171,0 \cdot 112)^{\prime}, \hat{\tau}_{3}=(-0 \cdot 606,0 \cdot 365,0 \cdot 193,0 \cdot 118)^{\prime}$. Under $H_{0}, Q(\tau C) \sim U_{4,2,30}$. The statistic $Q=0.82$, and the statistic $F_{3}=0.704<F_{8,54}(0 \cdot 95)=2 \cdot 097$. Thus the null hypothesis is accepted at the 5 per cent level, which means that the three standard lots are similar.

Finally, the equivalence between the test lot and the three standard lots is performed when $\delta$ is set to be $0 \cdot 2$. We found that the posterior odds is about 4 with $\pi\left(H_{0}\right)=\pi\left(H_{1}\right)=\frac{1}{2}$. We thus conclude that they are not equivalent.

\section{DISCUSSION}

The variance of the dissolution ratio, $R(t)$, increases with time. There are two methods to handle this situation. One is to model covariance structure allowing for different variances corresponding to different measurement times or model the variance as an increasing function of time. The other is to transform it to achieve stability, then use some simple structure to model the transformed series, for example AR(1) or uniform etc. Here we adopted the transformation method. Since the log transformation is a special case of the Box-Cox transformation defined in (1), the global maximum likelihood estimate of $\lambda$ should be close to 0 if $\log$ function gives a better performance. However, the estimate of $\lambda$ from our analysis is about $0 \cdot 7$, and the variance is not very stable as time increases, especially in the last measurement. If the time is cut into three pieces, $1-3,4-6,8-10$, just as in the design matrix in the beginning of the previous section, and the Box-Cox transformation is applied to the three pieces separately, the variances are more stabler and the estimates of the three power transformation parameters are $(-0.179,-0.553,-0.458)$, respectively. However, the prediction performance is not better, perhaps due to the increase in the number of parameters by two. Another question is the model selection. Table III compares the prediction performance in terms of MARD. It is clear that the conditional prediction approach is reasonable for $p_{2} \leqslant 4$. It is also more appropriate than the likelihood ratio test. However, when the number of measurement, $p$, changes, $p_{2}$ also changes. It is not clear what is an appropriate $p_{2}$ in general. Our suggestion is to do simulation to get some clues regarding the size of $p_{2}$. 

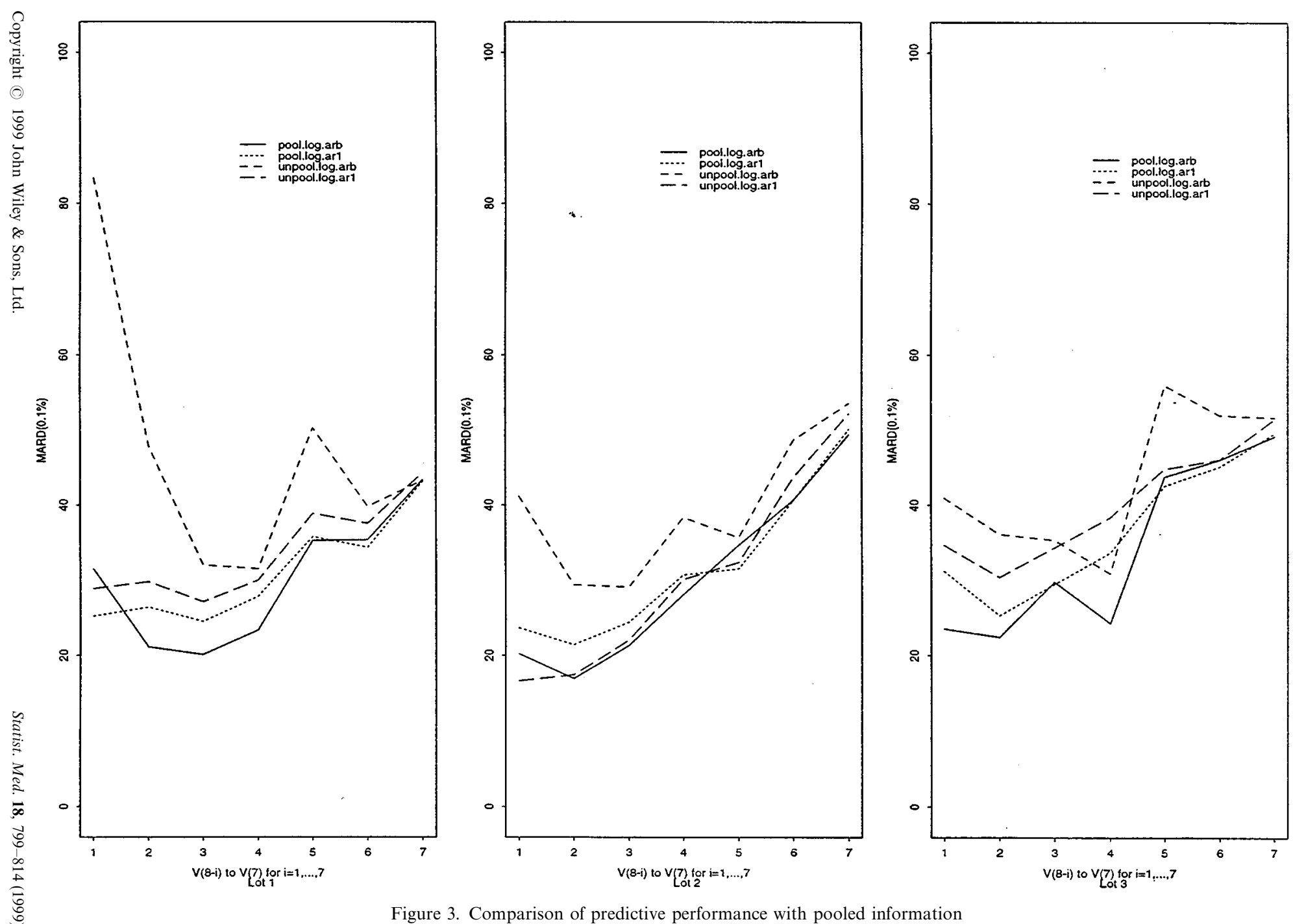

Figure 3. Comparison of predictive performance with pooled information 
Table VI. $\tau$ estimates for each batch

\begin{tabular}{|c|c|c|c|c|}
\hline$\hat{\tau}$ & Intercept & $\alpha_{1}$ & $\alpha_{2}$ & $\alpha_{3}$ \\
\hline Lot 1 & -0.564 & 0.362 & $0 \cdot 179$ & $\begin{array}{l}0 \cdot 174 \\
0 \cdot 113\end{array}$ \\
\hline Standard error & $0 \cdot 126$ & $0 \cdot 033$ & $0 \cdot 021$ & $0 \cdot 113$ \\
\hline $\begin{array}{l}\text { Lot } 2 \\
\text { Standard error }\end{array}$ & $\begin{array}{r}-0 \cdot 570 \\
0 \cdot 180\end{array}$ & $\begin{array}{l}0 \cdot 348 \\
0 \cdot 045\end{array}$ & $\begin{array}{l}0 \cdot 171 \\
0 \cdot 020\end{array}$ & $\begin{array}{l}0 \cdot 112 \\
0 \cdot 054\end{array}$ \\
\hline $\begin{array}{l}\text { Lot } 3 \\
\text { Standard error }\end{array}$ & $\begin{array}{r}-0 \cdot 606 \\
0 \cdot 129\end{array}$ & $\begin{array}{l}0 \cdot 365 \\
0 \cdot 043\end{array}$ & $\begin{array}{l}0 \cdot 193 \\
0 \cdot 036\end{array}$ & $\begin{array}{l}0 \cdot 118 \\
0 \cdot 091\end{array}$ \\
\hline $\begin{array}{l}\text { Combined losts ( } 1-3 \text { lots }) \\
\text { Standard error }\end{array}$ & $\begin{array}{r}-0.575 \\
0 \cdot 169\end{array}$ & $\begin{array}{l}0 \cdot 357 \\
0 \cdot 046\end{array}$ & $\begin{array}{l}0 \cdot 180 \\
0 \cdot 030\end{array}$ & $\begin{array}{l}0 \cdot 143 \\
0 \cdot 094\end{array}$ \\
\hline $\begin{array}{l}\text { Test lot } \\
\text { Standard merror }\end{array}$ & $\begin{array}{r}-1.069 \\
0.073\end{array}$ & $\begin{array}{l}0 \cdot 519 \\
0 \cdot 030\end{array}$ & $\begin{array}{l}0 \cdot 275 \\
0.032\end{array}$ & $\begin{array}{l}0.283 \\
0.105\end{array}$ \\
\hline
\end{tabular}

\section{APPENDIX: SOME DETAILS OF THE GENERAL GROWTH CURVE MODEL}

Let

$$
Y^{(\lambda)}=X \tau A+\varepsilon
$$

where $\tau=\left(\tau_{1}, \tau_{2}, \ldots, \tau_{r}\right)$ with $\tau_{i}$ representing the $i$ th group's mean function parameters, $r$ is the number of group, $X$ is a known $p \times m$ design matrix characterizing the type of polynomial for the regression function and $A$ a group indicator matrix. The ranks of $X$ and $A$ are $m<p$ and $r<n$, respectively. Also, $N=N_{1}+N_{2}+\ldots+N_{r}$, where $N_{i}$ is the number of vector observations in the $i$ th group. For example, if there is only one group, and the mean function is linear in time, then

$$
X=\left(\begin{array}{cccc}
1, & 1, & \ldots, & 1 \\
t_{1}, & t_{2}, & \ldots, & t_{p}
\end{array}\right)^{\prime}
$$

and $A=(1,1, \ldots, 1)$. If there are two groups, with $N_{1}$ of them in group 1 and $N_{2}$ in group 2 , then

$$
A=\left(\begin{array}{l}
1, \ldots, 1,0, \ldots, 0 \\
0, \ldots, 0,1, \ldots, 1
\end{array}\right)
$$

that is, there are $N_{1}(1,0)^{\prime} \mathrm{s}$ and $N_{2}(0,1)^{\prime} \mathrm{s}$ in $A$. Furthermore, $\varepsilon=\left(\varepsilon_{1}, \ldots, \varepsilon_{N}\right)$, where $\varepsilon_{i}$ are identically and independently distributed as $\mathrm{N}(0, \Sigma)$. The covariance matrix $\Sigma$ can be arbitrary positive definite or with some structures. Also, $v=0$ if $Y_{i j}^{\prime} s$ are positive. For the general growth curve model as given in (2), it is an extension of the growth curve model of Potthoff and Roy, ${ }^{2}$ having the Box-Cox transformation applied to the observation matrix $Y$.

The maximum likelihood estimates of $\lambda, \tau, \Sigma$ are given below:

$$
\hat{\tau}(\hat{\Sigma})=\left(X^{\prime} \hat{\Sigma}^{-1} X\right)^{-1} X^{\prime} \hat{\Sigma}^{-1} Y^{(\hat{\lambda})} A^{\prime}\left(A A^{\prime}\right)^{-1}
$$

and $\hat{\lambda}, \hat{\Sigma}$ maximize the profile likelihood function

$$
\begin{aligned}
L_{\max }(\lambda, \Sigma)= & |\Sigma|^{-\frac{N}{2}} \exp \left\{-\frac{1}{2} \operatorname{tr}\left(X^{\prime} \Sigma^{-1} X\right)^{-1} X^{\prime} \Sigma^{-1} S_{Y} \Sigma^{-1} X\right. \\
& \left.-\frac{1}{2} \operatorname{tr}\left(Z^{\prime} \Sigma Z\right)^{-1} Z^{\prime} Y^{(\lambda)} Y^{(\lambda)^{\prime}} Z\right\} \times \prod_{i, j}\left(Y_{i j}\right)^{(\lambda-1)} .
\end{aligned}
$$

The above representation is useful when $\Sigma$ has a certain structure. 
When $\Sigma$ is assumed arbitrary positive definite, the maximum likelihood estimates of $\tau$ and $\Sigma$ are

$$
\begin{aligned}
\hat{\tau} & =\left(X^{\prime} S^{-1} X\right)^{-1} X^{\prime} S^{-1} Y^{(\hat{\lambda})} A^{\prime}\left(A A^{\prime}\right)^{-1} \\
\hat{\Sigma} & =N^{-1}\left(Y^{(\hat{\lambda})}-X \hat{\tau} A\right)\left(Y^{(\hat{\lambda})}-X \hat{\tau} A\right)^{\prime} \\
S & =Y^{(\hat{\lambda})}\left(I-A^{\prime}\left(A A^{\prime}\right)^{-1} A\right) Y^{(\hat{\lambda}) \prime}
\end{aligned}
$$

and $\hat{\lambda}$ is obtained by maximizing the log profile likelihood function

$$
l_{\max }(\lambda)=-\frac{N}{2} \log |\hat{\Sigma}(\lambda)|+(\lambda-1) \cdot \sum_{i, j} \log \left(Y_{i j}\right) .
$$

It is noted that $\lambda$ can assume different values for different time intervals and (4) can be modified by writing the second term as a sum of several $\lambda$, that is, $\sum_{a=1}^{k}\left(\lambda_{a}-1\right) \Sigma_{i, j} \log \left(Y_{i j}\right)$, if there are $k$ intervals with $k$ different values of $\lambda$.

For the AR(1) covariance structure, $\Sigma=\sigma^{2} C, C=\left(\rho^{|i-j|}\right)$, Keramidas and Lee ${ }^{10}$ showed that the maximum likelihood estimates of $\tau, \sigma$ and $\lambda$ are given by

$$
\begin{aligned}
\hat{\tau} & =\left(X^{\prime} \hat{C}^{-1} X\right)^{-1} X^{\prime} \hat{C}^{-1} Y^{(\hat{\lambda})} A^{\prime}\left(A A^{\prime}\right)^{-1} \\
\hat{\sigma}^{2} & =\frac{1}{p N}\left(\operatorname{tr}\left(X^{\prime} \hat{C}^{-1} X\right)^{-1} X^{\prime} \hat{C}^{-1} S \hat{C}^{-1} X+\operatorname{tr}\left(Z^{\prime} \hat{C} Z\right)^{-1} Z^{\prime} Y^{(\hat{\lambda})} Y^{(\hat{\lambda})^{\prime}} Z\right)
\end{aligned}
$$

where $Z$ is a $p \times(p-m)$ matrix with rank $p-m$ such that $X^{\prime} Z=0, \hat{C}=\left(\hat{\rho}^{|i-j|}\right)$ and $\hat{\rho}, \hat{\lambda}$ are obtained by maximizing the $\log$ profile likelihood function

$$
l_{\max }(\rho, \lambda)=-\frac{p N}{2} \log \hat{\sigma}^{2}(\rho, \lambda)-\frac{N(p-1)}{2} \log \left(1-\rho^{2}\right)+(\lambda-1) \sum_{i, j} \log \left(Y_{i j}\right) .
$$

Comments regarding the $\lambda$ values for (4) are also applied here when there are different values of $\lambda$ for different time intervals.

The likelihood ratio criterion for testing $\operatorname{AR}(1)$ versus positive definite $\Sigma$ is

$$
\Lambda=N^{-\frac{p N}{2}}\left(\widehat{\sigma^{2}}(\rho)\right)^{\frac{-p N}{2}}\left(1-\rho^{2}\right)^{\frac{-N(p-1)}{2}}\left|\left(B^{\prime} Z\right)\right|^{-N}\left|Z^{\prime} Y Y^{\prime} Z\right|^{\frac{N}{2}}\left|X^{\prime} S^{-1} X\right|^{-\frac{N}{2}}
$$

with $B=\left(X^{\prime} X\right)^{-1} X^{\prime}$.

The conditional predictor of $V^{(2)}$ given $V^{(1)}$ is

$$
\hat{V}^{(2)}=X^{(2)} \hat{\tau} F+\hat{\Sigma}_{21} \hat{\Sigma}_{11}^{-1}\left(V^{(1)}-X^{(1)} \hat{\tau} F\right)
$$

where

$$
X=\left(\begin{array}{l}
X_{p_{1} \times m}^{(1)} \\
X_{p_{2} \times m}^{(2)}
\end{array}\right)
$$

$F$ is a known $r \times 1$ group indicator vector, $\hat{\tau}$ and

$$
\hat{\Sigma}=\left(\begin{array}{ll}
\hat{\Sigma}_{11} & \hat{\Sigma}_{12} \\
\hat{\Sigma}_{21} & \hat{\Sigma}_{22}
\end{array}\right)
$$

are the maximum likelihood estimates of $\tau$ and $\Sigma$.

We next consider some testing problem for the Potthoff and $\mathrm{Roy}^{2}$ in which no Box-Cox transformation is applied to the observation matrix $Y$. In order to test the null hypothesis $H_{0}: \tau=\tau_{0}$ against the alternative hypothesis $H_{1}: \tau \neq \tau_{0}$, Khatri ${ }^{7}$ showed that for general $r$, 
under $H_{0}$

$$
Q\left(\tau_{0}\right)=\frac{\left|\left(X^{\prime} S^{-1} X\right)^{-1}\right|}{\left|\left(X^{\prime} S^{-1} X\right)^{-1}+\left(\tau-\tau_{0}\right) G\left(\tau-\hat{\tau}_{0}\right)^{\prime}\right|} \sim U_{m, r, N-r-p+m}
$$

where $G=A A^{\prime}-A Y^{\prime} Z\left(Z^{\prime} Y Y^{\prime} Z\right)^{-1} Z^{\prime} Y^{\prime} A^{\prime}, \hat{\tau}$ and $S$ are given in (3) with $Y^{(\hat{\lambda})}$ replaced by $Y$. When $r=1$, the test statistic becomes

$$
F_{1}=\frac{N-p}{m} G\left(\hat{\tau}-\tau_{0}\right)^{\prime}\left(X^{\prime} S^{-1} X\right)\left(\hat{\tau}-\tau_{0}\right) \sim F_{m, N-p} .
$$

As for the $r$ sample test, we have, under $H_{0}$

$$
Q(\tau D=0)=\frac{\left|\left(X^{\prime} S^{-1} X\right)^{-1}\right|}{\left|\left(X^{\prime} S^{-1} X\right)^{-1}+\hat{\tau} D\left(D^{\prime} G^{-1} D\right)^{-1} D^{\prime} \hat{\tau}^{\prime}\right|} \sim U_{m, k, N-r-p+m}
$$

where $G^{-1}=\left(A A^{\prime}\right)^{-1}+\left(A A^{\prime}\right)^{-1} A Y^{\prime} Z\left(Z^{\prime} S Z\right)^{-1} Z^{\prime} Y A^{\prime}\left(A A^{\prime}\right)^{-1}$.

When $m=1,2$ or $q=1,2$, special results are available, see Anderson. ${ }^{11}$ For example, when $q=1$

$$
F_{2}=\left(\frac{1}{U_{1, m, N-1-p}}-1\right) \times \frac{N-1-p}{m} \sim F_{m, N-1-p} .
$$

When $q=2$

$$
F_{3}=\frac{\left(1-\sqrt{ } U_{m, 2, n}\right)}{\sqrt{ } U_{m, 2, n}} \times \frac{n-1-m}{m} \sim F_{2 m, 2(n+1-m)} .
$$

For the situation where special results are not available, we can use the following approximation:

$$
F_{4}=-\left\{N-r-p+m-\frac{1}{2}(m-q+1)\right\} \log (Q) / \chi^{2}(0 \cdot 95)
$$

by Pearson's type I distribution. The critical values are given in Krishnaiah and Lee. ${ }^{8}$

\section{ACKNOWLEDGEMENTS}

The authors thank the referees and an editor for some constructive comments. They are also grateful for research support from the National Science Council of Taiwan (87-2119-M-009-001 and 86-2155-M-009-037-T).

\section{REFERENCES}

1. Chen, J. and Tsong, Y. 'Multiple time-point dissolution specification for a sampling acceptance plan', Journal of Biophysical Statistics, 7, 259-270 (1997).

2. Potthoff, R. F. and Roy, S. N. 'A generalized multivariate analysis of variance model useful especially for a growth curve problem', Biometrika, 51, 313-326 (1964).

3. Lee, J. C. 'Tests and model selection for the general growth curve model', Biometrics, 47, 147-159 (1991).

4. Geisser, S. C. 'The predictive sample reuse method with application', Journal of the American Statistical Association, 70, 320-328 (1975).

5. Lee, J. C. and Geisser, S. 'Growth curve prediction', Sankhya, Series A, 34, 393-412 (1972).

6. Keramidas, E. M. and Lee, J. C. 'Selection of a covariance structure for growth curves', Biomedical Journal, 37, 783-797 (1995) 
7. Khatri, C. G. 'A note on MANOVA model applied to problems in growth curve', Annals of the Institute of Statistical Mathematica, 18, 75-86 (1966).

8. Krishnaiah, P. R. and Lee, J. C. 'Likelihood ratio tests for mean vectors and covariance matrices', in Handbook of Statistics, Vol. I, 1980, pp. 513-570.

9. Hung, H. N. and Wong, W. H. 'Average likelihood', Technical Report No. 429, Department of Statistics, The University of Chicago, 1996.

10. Keramidas, E. M. and Lee, J. C. 'Forecasting technological substitutions with concurrent short time series', Journal of the American Statistical Association, 85, 625-632 (1990).

11. Anderson, T. W. An Introduction to Multivariate Statistical Analysis, 2nd edn, Wiley, New York, 1984. 